\title{
Can MRI Observations Predict Treatment Outcome of Lavage in Patients with Painful TMJ Disc Displacement without Reduction?
}

\author{
EwaCarin Ekberg ${ }^{1}$, Lars-Göran Hansson ${ }^{2}$, Thomas List $^{1}$, Lars Eriksson ${ }^{3}$, Lotta Englesson \\ Sahlström ${ }^{4}$, Arne Petersson ${ }^{5}$ \\ ${ }^{1}$ Department of Orofacial Pain and Jaw Function, Faculty of Odontology, Malmö University, Sweden. \\ ${ }^{2}$ Department of Dento Maxillofacial Radiology, Center for Medical Imaging and Physiology, Skåne University Hospital, \\ Lund, Sweden. \\ ${ }^{3}$ Department of Oral Surgery and Oral Medicine, Faculty of Odontology, Malmö University, Malmö, Sweden. \\ ${ }^{4}$ Department of Oral and Maxillofacial Surgery, Skåne University Hospital, Lund, Sweden. \\ ${ }^{5}$ Department of Oral and Maxillofacial Radiology, Faculty of Odontology, Malmö University, Malmö, Sweden.
}

\author{
Corresponding Author: \\ Arne Petersson \\ Department of Oral and Maxillofacial Radiology, Faculty of Odontology \\ Malmö University \\ SE-20506 Malmö \\ Sweden \\ Phone: 0046706709745 \\ Fax: 0046406657010 \\ E-mail: arne.petersson@mah.se
}

\begin{abstract}
Objectives: The purpose of this study was to examine magnetic resonance imaging findings in patients with painful disc displacement without reduction of the temporomandibular joint to determine whether the findings were able to predict treatment outcome of lavage and a control group treated with local anaesthesia without lavage in a short-term: 3-month perspective.

Material and Methods: Bilateral magnetic resonance images were taken of 37 patients with the clinical diagnosis of painful disc displacement without reduction. Twenty-three patients received unilateral extra-articular local anaesthetics and 14 unilateral lavage and extra-articular local anaesthetics. The primary treatment outcome defining success was reduction in pain intensity of at least $30 \%$ during jaw movement at the 3-month follow-up.

Results: Bilateral disc displacement was found in 30 patients. In 31 patients the disc on the treated side was deformed, and bilaterally in 19 patients. Osteoarthritis was observed in 28 patients, and 13 patients had bilateral changes. Thirty patients responded to treatment and 7 did not, with no difference between the two treated groups. In neither the treated nor the contralateral temporomandibular joint did treatment outcome depend on disc diagnosis, disc shape, joint effusion, or osseous diagnoses. Magnetic resonance imaging findings of disc position, disc shape, joint effusion or osseous diagnosis on the treated or contralateral side did not give information of treatment outcome.

Conclusions: Magnetic resonance imaging findings could not predict treatment outcome in patients treated with either local anaesthetics or local anaesthetics and lavage.
\end{abstract}

Keywords: magnetic resonance imaging; pain; temporomandibular joint; temporomandibular joint disc; therapeutic irrigation.

Accepted for publication: 10 February 2015

To cite this article:

Ekberg E, Hansson LG, List T, Eriksson L, Sahlström LE, Petersson A. Can MRI Observations Predict Treatment Outcome of Lavage in Patients with Painful TMJ Disc Displacement without Reduction?

J Oral Maxillofac Res 2015;6(1):e5

URL: http://www.ejomr.org/JOMR/archives/2015/1/e5/v6n1e5.pdf

doi: $10.5037 /$ jomr.2015.6105 


\section{INTRODUCTION}

Lavage of the temporomandibular joint (TMJ) is a minimally invasive treatment modality with a low rate of side effects and has been recommended for patients with painful disc displacement without reduction (DDwoR) [1]. This pain, when it becomes chronic, often leads not only to protracted personal suffering and reduced quality of life but also to extensive financial costs for society. However, no consistent evidence supports or refutes use of arthrocentesis and lavage in TMJ disorders [를 Recently, we performed a single-blind randomised controlled trial (RCT) and it showed that lavage did neither improve pain nor mouth opening capacity better than extra-articular local anaesthetics in patients with painful DDwoR [ $\underline{4}$.

The diagnosis DDwoR is usually based on clinical findings, but it has been shown that diagnoses set clinically and found on magnetic resonance imaging (MRI) do not always correspond [5-7]. Because clinical and MRI diagnoses of silent joints can differ $[\underline{5}, \underline{6}]$, MRI is an important aid in establishing the correct position of the TMJ disc. Other findings such as hard tissue changes and joint effusion can also be found on MRI of the TMJ.

Prognostic factors for the success or failure of lavage to relieve pain have been the subject of many investigations, with conflicting results [8-13]. Diagnoses of internal derangement and osteoarthritis have not been found to be linked to the outcome of arthrocentesis regarding TMJ pain and mandibular range of motion [9]. This agrees with the observations of Smolka and Iizuka [11], who found that preoperative arthroscopic findings did not seem to correlate with treatment outcome. A reduced pain-free mouth opening $(<30 \mathrm{~mm})$ before treatment together with MRI confirmation of a stuck disc and unchanged disc shape during mouth opening is reported to be a prognostic factor for no response to lavage [10]. Patients with TMJ effusion have been found to respond less favourably to arthrocentesis and hydraulic distention [12]. Honda et al. [13] concluded that the outcome of arthrocentesis treatment is not influenced by disc configuration, amount of disc displacement, or range of disc motion at mouth opening but by amount of joint fluid: patients with no or minimal joint fluid had a good response to treatment compared to patients with extensive joint effusion. Sato et al. []ㅡ, however, found no correlation between joint effusion and the clinical condition of patients before and after arthroscopic lysis and lavage of the TMJ. In addition, Honda et al. [13] found joints with erosive cortical changes to be associated with pain after arthrocentesis. These conflicting results made it interesting to study MRI findings on influences in treatment outcome of TMJ lavage. This article presents additional results of our study on TMJ lavage [4].

The purpose of this study was to determine whether magnetic resonance imaging findings in patients with painful disc displacement without reduction of the temporomandibular joint are able to predict treatment response to lavage.

Hypothesis: MRI findings does not predict treatment outcome of lavage in patients with painful DDwoR.

\section{MATERIAL AND METHODS Participants}

In this study 55 patients were included according to inclusion and exclusion criteria described in the RCT by Sahlström et al. [4]. Exclusion criteria were: diagnosed systemic muscular or joint disease; a whiplash-associated disorder; difficulties with the Swedish language; dental causes for the pain; tumours of the TMJ; disturbed coagulation ability; pacemaker or other contraindications for MRI; and pregnancy. Ten patients were excluded due to one or more of the exclusion criteria. Overall, forty-five patients (41 women and 4 men, 34.9 [14.2] years) participated. All patients were recruited between June 2003 and November 2007. At the 3-month follow-up, 8 patients had dropped out, resulting in 37 remaining patients (Figure 1).

The Regional Ethics Review Board at Lund University approved the study (Daybook no. [Dnr] LU 109-03), and all patients signed an informed-consent form. Patients received no monetary compensation.

\section{Study design}

At baseline the patients completed a questionnaire before the clinical and radiographic examination. The clinical examination was performed according to research diagnostic criteria (RDC/TMD) by an experienced orofacial pain specialist (ECE) calibrated in RDC/TMD examination methods [14]. The radiographic examination comprised: (i) panoramic radiographs to exclude dental causes for the pain and (ii) MR images to verify the clinical diagnosis of DDwoR. A diagnosis of DDwoR was set if the RDC/TMD indicated DDwoR and MRI confirmed DDwoR [14]. One examiner, blinded to patient treatment, evaluated the patients at baseline and at 3 months after intervention. Pre-treatment and 3-month follow-up 


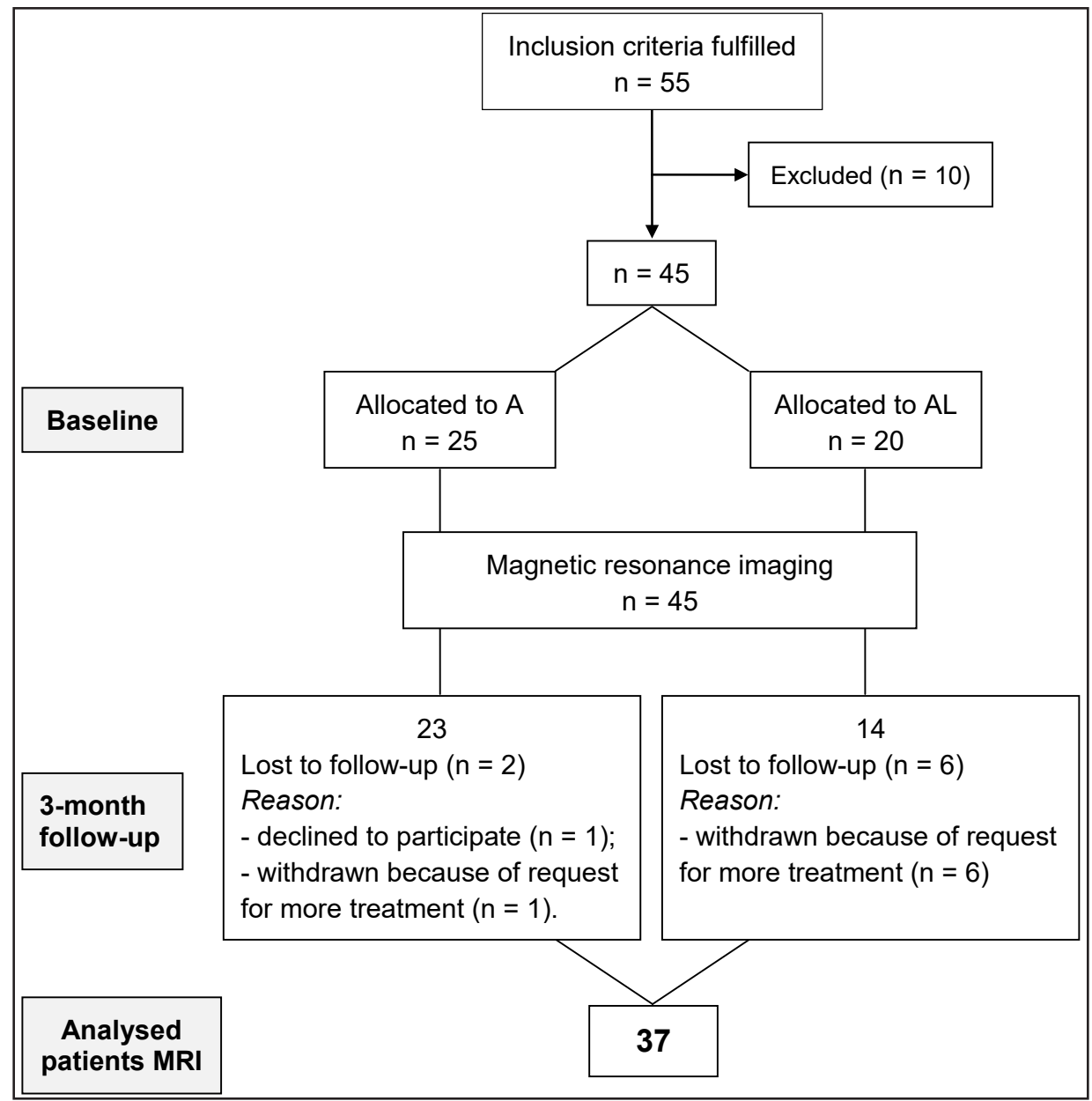

Figure 1. Distribution and flow chart of patients with temporomandibular joint disc displacement without reduction and temporomandibular disorders pain at baseline and at the 3-month follow-up. $\mathrm{A}=$ treatment with extra-articular local anaesthetics; $\mathrm{AL}=$ treatment with extraarticular local anaesthetics and lavage; $\mathrm{n}=$ number of patients; MRI = magnetic resonance imaging.

assessments involved a standardised evaluation of signs and symptoms of TMD [4]. Clinical assessments in this study included:

1. Characteristic pain index (CPI): The CPI describes the mean value of current, worst, and average TMD-related pain during the last 6 months rated on a $0-10$ NRS [14].

2. Mandibular range of motion: The vertical range of motion of the mandible was determined by measuring unassisted opening without pain and maximum assisted opening in $\mathrm{mm}$ according to the RDC/TMD protocol [14].

3. Treatment responders: The primary outcome measure of the study was pain relief during jaw movement of at least $30 \%$ on the visual analogue scale (VAS) [15].

\section{Magnetic resonance imaging}

In all patients MRI of the TMJ was performed bilaterally. A Siemens Magnetom Vision MRI machine was used between 2003 and June 2004 and a Siemens Magnetom Sonata Vision between July 2004 and December 2007 (Siemens, Erlangen, Germany). Both were 1.5 Tesla machines. MR images were taken with a bilateral TMJ surface coil. Images were taken in the closed and open mouth positions. In the closed mouth position, patients closed their mouth with their teeth in contact; sagittal and coronal images of the TMJ were taken. In the open mouth position, patients opened their mouths as much as possible without experiencing unbearable pain and a stepped plastic bite-block was placed between the upper and lower incisors; sagittal images of the TMJ were taken. Sagittal sections were orientated perpendicular to the long axis of the condyle, and coronal sections were taken parallel to the long axis. The orientation was done through axial localisers, one for the closed mouth position and one for the open mouth position. Proton density (PD) and T2 weighted images were acquired using a double-echo turbo spin-echo sequence, $\mathrm{TE}=15 / 105 \mathrm{~ms}, \mathrm{TR}=2400$ (sagittal) or 2000 (coronal), turbo factor $=7, \mathrm{FOV}=160 \mathrm{x}$ $160 \mathrm{~mm}^{2}$, matrix $=255 \times 512$ and slice thickness $3 \mathrm{~mm}$. Eight to ten sections were obtained for each sequence. 
Two experienced oral radiologists (L-GH and AP) interpreted the MR images blinded, with no knowledge of patients' clinical diagnoses. Observer calibration and subsequent image assessment occurred as follows: The observers interpreted the first 10 complete MRI examinations together. Each observer then interpreted the remaining 35 examinations separately. Observer assessments of these 35 examinations were compared, and if they differed, findings were re-evaluated and discussed until a consensus was reached.

The interpretation of the MR images followed the criteria given by Ahmad et al. [16] included in the RDC/TMD validation project. Diagnoses of disc position (Table 1) and osseous changes (Table 2) were made according to Ahmad et al. [16].

\section{Randomisation}

Patients were randomised to one of two treatment options:

- Group A - local anaesthetics;

- Group AL - local anaesthetics and lavage.

The randomisation procedure is described in detail by Sahlström et al. [4].

\section{Treatment}

One surgeon (LES) with 5 years of surgical experience performed all treatment procedures. This surgeon did not evaluate treatment outcome. The duration of each treatment was similar.

Directly preceding treatment, patients were anesthetized with $2.5 \mathrm{ml}$ Xylocain-adrenalin ${ }^{\circledR}$ (ASTRA Zeneca) $20 \mathrm{mg} / \mathrm{ml}$ for auriculotemporalis block.
In Group A, saline was flushed into a cup that was hidden from the patient before the drape was removed to simulate lavage. In the $\mathrm{AL}$ group, a cannula was placed in the posterior part of the upper joint compartment. A second, wider cannula was then inserted in the same compartment. Drainage was tested by carefully injecting a small amount of saline. The joint was then flushed with $50 \mathrm{ml}$ saline. Excess saline was evacuated by aspiration after the procedure.

\section{Statistical analysis}

Inter-observer agreement was assessed by calculating the kappa value between observers L-GH and AP on disc position, disc shape, joint effusion, and osteoarthritis according to kappa statistics (Kappa $\leq 0.20$, poor; $0.21-0.4$, fair; $0.41-0.6$, moderate; 0.61 - 0.8, good; $0.81-1$, very good agreement) [17].

The results were presented as mean and standard deviation (M [SD]). Differences in categorical variables between Group A and AL; and treatment responders and non-responders were analysed with the chi-square test and Fisher's exact test. All inferential statistical tests were two-tailed with a significant level of $\mathrm{P} \leq 5 \%$. Statistical analyses were done with the Statistical Package for the Social Sciences (SPSS, ver. 18.0 for Windows; SPSS Inc., IL, USA).

\section{RESULTS}

Thirty-seven patients with painful disc displacement without reduction treated with local anaesthetics (Group A, $\mathrm{n}=23$ ) or local anaesthetics and lavage (Group AL, $\mathrm{n}=14$ ) participated in the 3-month follow-up (Figure 1). MRI findings were analysed in 34 women and 3 men; mean ages were 35.7 (15.8) years in Group A and 35.6 (12.9) years in Group AL.

Table 1. Disc diagnosis for the TMJ according to Ahmad et al. [16]

A. Normal: Disc location is normal on closed- and open-mouth images.

B. Disc displacement with reduction: Disc location is displaced on closed-mouth images but normal in open-mouth images.

C. Disc displacement without reduction: Disc location is displaced on closed-mouth and open-mouth images.

D. Indeterminate: Disc location is not clearly normal or displaced in the closed-mouth position.

E. Disc not visible: Neither signal intensity nor outlines make it possible to define a structure as the disc in the closed-mouth and openmouth views. If the images are of adequate quality in visualizing other structures in the TMJ, then this finding is interpreted to indicate a deterioration of the disc, which is associated with advanced disc pathology.

Table 2. Osseous diagnosis of the temporomandibular joint according to Ahmad et al. [16]

A. No osteoarthritis: No subcortical sclerosis or articular surface flattening; and no deformation due to subcortical cyst, surface erosion, osteophyte, or generalized sclerosis.

B. Indeterminate for osteoarthritis: Subcortical sclerosis with/without articular surface flattening; or articular surface flattening with/ without subcortical sclerosis; and no deformation due to subcortical cyst, surface erosion, osteophyte, or generalized sclerosis.

C. Osteoarthritis: Deformation due to subcortical cyst, surface erosion, osteophyte, or generalized sclerosis. 
Out of 37 patients 30 were responders to treatment (Group A: 19, Group AL: 11) and 7 non-responders (Group A: 4, Group AL: 3), with no significant difference on treatment response between Group A and AL [4]. For a more detailed description of the results, see Sahlström et al. [4].

\section{Clinical and self-report measures}

Differences at baseline in pain intensity between the two treatment groups calculated as CPI and mouth opening capacity without pain as well as assisted opening capacity were non-significant (Table 3). Mandibular range of motion, and pain intensity were not predictors of treatment response.

\section{Inter-observer agreement}

The kappa value was moderate for disc shape (0.55) and joint effusion (0.55), and good for osteoarthritis $(0.65)$ and disc position $(0.75)$.

\section{Differences in MRI findings between Group A and $\mathrm{AL}$}

No differences were found between Group A and AL regarding the frequency of disc displacement $(\mathrm{P}=1)$, disc shape $(\mathrm{P}=1)$, joint effusion $(\mathrm{P}=0.414)$, and hard tissue changes $(\mathrm{P}=1)$ (Table 3$)$.

The groups $\mathrm{A}$ and $\mathrm{AL}$ were merged when prognostic factors of MRI findings were analysed.
Table 3. Baseline characteristics and MRI findings at baseline in group A and group AL

\begin{tabular}{|c|c|c|}
\hline Clinical and MRI findings & $\begin{array}{c}\text { Group A } \\
(\mathbf{n}=\mathbf{2 3})\end{array}$ & $\begin{array}{c}\text { Group AL } \\
(\mathrm{n}=14)\end{array}$ \\
\hline \multicolumn{3}{|c|}{ Mouth opening in mm (Mean [SD]) } \\
\hline Without pain & $28(1.7)$ & $27(1.4)$ \\
\hline Maximum assisted & $36(1.7)$ & $35(1.8)$ \\
\hline CPI (Mean [SD]) & $6.15(0.31)$ & $6.97(0.34)$ \\
\hline \multicolumn{3}{|c|}{ Disc displacement without reduction } \\
\hline Unilateral & 15 & 9 \\
\hline Bilateral & 8 & 5 \\
\hline \multicolumn{3}{|l|}{ Disc deformation } \\
\hline Unilateral & 11 & 7 \\
\hline Bilateral & 10 & 6 \\
\hline \multicolumn{3}{|l|}{ Joint effusion } \\
\hline Unilateral & 7 & 8 \\
\hline Bilateral & 7 & 3 \\
\hline \multicolumn{3}{|l|}{ Hard tissue changes } \\
\hline Unilateral & 10 & 7 \\
\hline Bilateral & 7 & 5 \\
\hline
\end{tabular}

\section{MRI findings as predictors of treatment outcome}

Treatment outcome did not depend on disc diagnosis, disc shape, joint effusion or osseous diagnosis in neither the treated nor the contralateral temporomandibular joint (Table 4).

Table 4. Magnetic resonance imaging (MRI) findings in treatment side and contralateral side related to responders and non-responders to treatment

\begin{tabular}{|c|c|c|c|c|}
\hline & $\begin{array}{c}\text { Treatment } \\
(\mathrm{n}=\mathbf{3 7} \text { joints })\end{array}$ & $\begin{array}{l}\text { Contralateral } \\
(\mathrm{n}=\mathbf{3 7} \text { joints })\end{array}$ & $\begin{array}{c}\text { Responders } \\
(\mathrm{n}=\mathbf{3 0} \text { patients })\end{array}$ & $\begin{array}{c}\text { Non-responders } \\
(\mathrm{n}=7 \text { patients })\end{array}$ \\
\hline \multicolumn{5}{|l|}{ Disc diagnosis } \\
\hline DDwoR (treatment) & 37 & & 30 & 7 \\
\hline DDwoR (contralateral) & & 13 & 12 & 1 \\
\hline DDwR (contralateral) & & 17 & 12 & 5 \\
\hline Normal (contralateral) & & 7 & 6 & 1 \\
\hline \multicolumn{5}{|l|}{ Disc shape } \\
\hline Normal & 6 & 18 & 5 & 1 \\
\hline Deformed & 31 & 19 & 25 & 6 \\
\hline \multicolumn{5}{|l|}{ Joint effusion } \\
\hline None & 14 & 25 & 12 & 2 \\
\hline Slight & 14 & 9 & 13 & 2 \\
\hline Frank & 9 & 3 & 5 & 3 \\
\hline \multicolumn{5}{|l|}{ Osseous diagnosis } \\
\hline No osteoarthritis & 9 & 24 & 6 & 2 \\
\hline Osteoarthritis & 28 & 13 & 24 & 5 \\
\hline
\end{tabular}

$\mathrm{n}=$ number of joints/patients; DDwoR = disc displacement without reduction; DDwR = disc displacement with reduction.

Numbers of patients are given for MRI findings. No significant differences were found between the groups in any of the measures. 


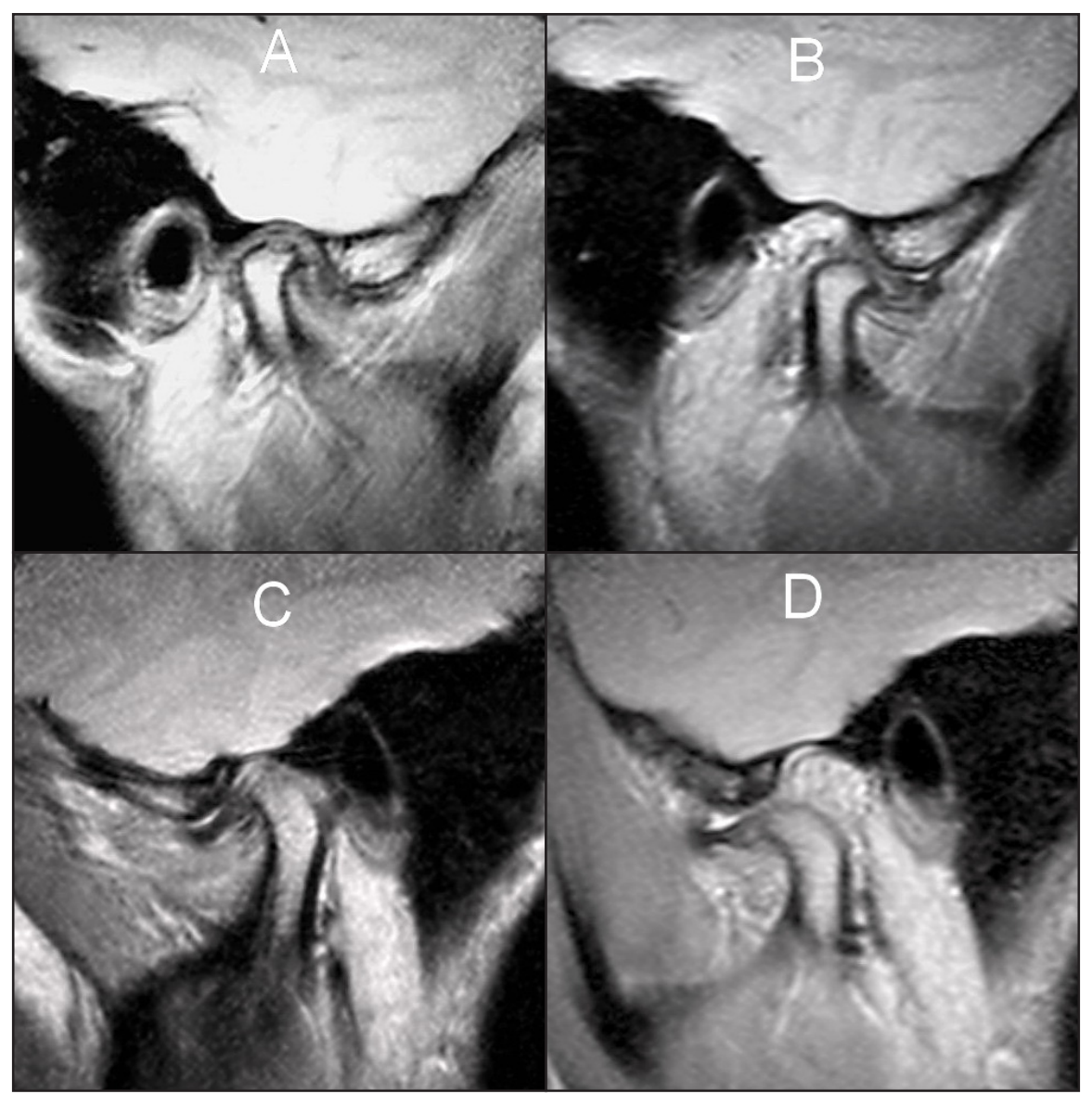

Figure 2. Proton density magnetic resonance images of the right ( $\mathrm{A}$ and $\mathrm{B})$ and left $(\mathrm{C}$ and $\mathrm{D})$ temporomandibular joint of the same patient. The left side was treated with lavage. The disc is displaced anteriorly bilaterally at closed-mouth (A and C) and open-mouth positions (B and D). The condylar movements at mouth opening are restricted. The diagnosis was set to bilateral anterior disc displacement without reduction. $\mathrm{A} /$ and $\mathrm{B} /$ right side at closed-mouth position (A) and at open-mouth position (B). Erosion on the superior surface of the condyle and a small osteophyte are visible.

$\mathrm{C}$ / and D/ Left side at closed-mouth position (C) and at open-mouth position (D). The condyle is flattened and a small osteophyte is visible.

Twenty-four of 30 treatment responders and 6 of 7 non-responders had bilateral disc displacement. Figure 2 illustrates a patient with bilateral disc displacement without reduction. The discs in 12 patients were displaced laterally in combination with disc displacement without reduction and 7 patients out of these were responders to treatment.

Hard tissue changes were found in 29 of the 37 patients. Osteoarthritis occurred in both responders and non-responders. Erosions were found in 14 patients, and 12 of these were on the treatment side. Of the 12 joints 11 were in treatment responders.

\section{DISCUSSION}

The hypothesis of this single-blind RCT that MRI findings does not predict treatment outcome of lavage in patients with painful DDwoR could not be rejected. In neither the treated nor the contralateral TMJ did treatment outcome depend on disc diagnosis, disc shape, joint effusion or osseous diagnoses.
These findings agree with other studies that report no correlation between MRI findings and treatment outcome $[\underline{9}, \underline{11}]$.

Diagnostic radiology is part of a larger system, the goal of which is to treat patients effectively and efficiently [17]. According to Fryback and Thornbury [17] the ultimate criterion for the usefulness of a diagnostic test from a patient viewpoint is whether it leads to a change in management that is beneficial for the patient. MRI is considered the most reliable and useful imaging modality for evaluating internal derangements and osteoarthritis of the TMJ [18]. A systematic review of the literature on the diagnostic efficacy of MRI concluded that none of the searched publications reported therapeutic efficacy or patient outcome efficacy and it was impossible to determine when an MRI examination would give information of the patient's treatment outcome [19]. We found that MRI information did not affect the ability to predict treatment outcome. So the role of MRI in the management of painful DDwoR is questionable. MRI may be a useful diagnostic tool in treatment planning, 
but it is not useful in the prediction of treatment outcome regarding lavage in patients with disc displacement without reduction.

The major strength of our study is that the observers were calibrated in the interpretation of the MR images and blinded to patients' clinical diagnoses and treatment. Another strength of this RCT is the singleblind design in the clinical setting. Further, the clinical examination followed RDC/TMD methods. Osseous changes were diagnosed using MRI. Ahmad et al. [16] recommended use of CT for detecting osseous changes because MRI has a lower sensitivity than CT in visualizing osseous changes. An underestimation of osseous changes may have occurred.

Emshoff and Rudisch [12] found joint effusion of the TMJ to be specific MRI finding related to unsuccessful treatment outcome of arthrocentesis in a group of 37 patients. The clinical trial of Honda et al. [13] confirmed these results and reported a better treatment outcome in 36 patients with no or minimal joint effusion compared to those with extensive amounts of effusion. These two studies contradict our results, and the discrepancy could be due to our use of a control group [4]. Sato et al. []] took MR images before and after arthroscopic lysis and lavage and found no correlation between joint effusion and the clinical condition after treatment.

Some discussion has considered erosive cortical changes in the condyle to be an accurate finding with a high impact for patients presenting with TMJ pain and joint sounds $[20,21]$. Such a pathologic lesion in the condylar head increases joint friction, and it is conceivable that risk of pain and dysfunction in the joint increases. Twelve joints (32\%) in our study had erosions on the condylar head in the treated joint and more than $90 \%$ of these had a positive treatment outcome regardless of treatment modality. This is not in line with Honda et al. [13], who reported that erosive condylar changes were significantly lower in the improved group. This disagreement may be due to differences in the timing of treatment outcome measurement. We followed our patients for 3 months, and Honda et al. [13] followed their patients for 6 weeks. Other differences in study design include a 3-week pre-treatment with non-steroidal anti-inflammatory drugs and lack of an RCT design in the Honda study.

Our second hypothesis, that we would find arthrosis and disc displacement in the contralateral untreated TMJ, could not be rejected. To our knowledge, the importance of MRI findings on the contralateral and untreated TMJ for the outcome of lavage, have not been previously studied. An autopsy study that microscopically evaluated structural changes of the disc, the condyle and the temporal component in 20 individuals reported similar findings in both TMJs [22]. Such structural changes on the contralateral untreated TMJ in our study had no impact on treatment outcome.

\section{CONCLUSIONS}

Treatment outcome did not depend on disc diagnosis, disc shape, joint effusion or osseous diagnosis in neither the treated nor the contralateral temporomandibular joint. Thus magnetic resonance imaging findings could not predict treatment outcome of local anaesthetics or local anaesthetics and lavage of painful disc displacement without reduction. The clinical implication of the study is that magnetic resonance imaging confirmation of a clinical diagnosis of disc displacement without reduction in patients treated with either local anaesthetics or local anaesthetics and lavage appears to be unnecessary.

\section{ACKNOWLEDGMENTS AND DISCLOSURE STATEMENTS}

The authors report no conflicts of interest related this study.

\section{REFERENCES}

1. Nitzan DW. Arthrocentesis--incentives for using this minimally invasive approach for temporomandibular disorders. Oral Maxillofac Surg Clin North Am. 2006 Aug;18(3):311-28, vi. [Medline: 18088835]

2. Guo C, Shi Z, Revington P. Arthrocentesis and lavage for treating temporomandibular joint disorders. Cochrane Database Syst Rev. 2009 Oct 7;(4):CD004973. [Medline: 19821335] [doi: 10.1002/14651858.CD004973.pub2]

3. Petersson A, Eriksson L, Lundh H. No short-term difference in outcome after temporomandibular joint arthrography alone or with immediate lavage. Oral Surg Oral Med Oral Pathol. 1994 Apr;77(4):322-6. [Medline: 8015793] [doi: 10.1016/0030-4220(94)90190-2]

4. Sahlström LE, Ekberg EC, List T, Petersson A, Eriksson L. Lavage treatment of painful jaw movements at disc displacement without reduction. A randomized controlled trial in a short-term perspective. Int J Oral Maxillofac Surg. 2013 Mar;42(3):356-63. [Medline: 23140985] [doi: 10.1016/j.ijom.2012.10.011] 
5. Barclay P, Hollender LG, Maravilla KR, Truelove EL. Comparison of clinical and magnetic resonance imaging diagnosis in patients with disk displacement in the temporomandibular joint. Oral Surg Oral Med Oral Pathol Oral Radiol Endod. 1999 Jul;88(1):37-43. [Medline: 10442943] [doi: 10.1016/S1079-2104(99)70191-5]

6. Limchaichana N, Nilsson H, Ekberg EC, Nilner M, Petersson A. Clinical diagnoses and MRI findings in patients with TMD pain. J Oral Rehabil. 2007 Apr;34(4):237-45. [Medline: 17371560] [doi: 10.1111/j.1365-2842.2006.01719.x]

7. Koh KJ, List T, Petersson A, Rohlin M. Relationship between clinical and magnetic resonance imaging diagnoses and findings in degenerative and inflammatory temporomandibular joint diseases: a systematic literature review. J Orofac Pain. 2009 Spring;23(2):123-39. [Medline: 19492537]

8. Sato J, Segami N, Kaneyama K, Nishimura M, Suzuki T. Importance of the changes in joint effusion shown by magnetic resonance imaging before and after arthroscopic lysis and lavage of the temporomandibular joint. Br J Oral Maxillofac Surg. 2002 Feb;40(1):72-5. [Medline: 11883976] [doi: 10.1054/bjom.2001.0737]

9. Emshoff R, Rudisch A. Are internal derangement and osteoarthrosis linked to changes in clinical outcome measures of arthrocentesis of the temporomandibular joint? J Oral Maxillofac Surg. 2003 Oct;61(10):1162-7; discussion 1167-70. [Medline: 14586851] [doi: 10.1016/S0278-2391(03)00676-1]

10. Iwase H, Sasaki T, Asakura S, Asano K, Mitrirattanakul S, Matsuka Y, Imai Y. Characterization of patients with disc displacement without reduction unresponsive to nonsurgical treatment: a preliminary study. J Oral Maxillofac Surg. 2005 Aug;63(8):1115-22. [Medline: 16094578] [doi: 10.1016/j.joms.2005.04.005]

11. Smolka W, Iizuka T. Arthroscopic lysis and lavage in different stages of internal derangement of the temporomandibular joint: correlation of preoperative staging to arthroscopic findings and treatment outcome. J Oral Maxillofac Surg. 2005 Apr;63(4):471-8. [Medline: 15789318] [doi: 10.1016/j.joms.2004.07.021]

12. Emshoff R, Rudisch A. Temporomandibular joint internal derangement and osteoarthrosis: are effusion and bone marrow edema prognostic indicators for arthrocentesis and hydraulic distention? J Oral Maxillofac Surg. 2007 Jan;65(1):66-73. [Medline: 17174766] [doi: 10.1016/j.joms.2005.11.113]

13. Honda K, YasukawaY, Fujiwara M,Abe T, Urade M. Causes of persistent joint pain after arthrocentesis of temporomandibular joint. J Oral Maxillofac Surg. 2011 Sep;69(9):2311-5. [Medline: 21664744] [doi: 10.1016/j.joms.2011.02.025]

14. Dworkin SF, LeResche L. Research diagnostic criteria for temporomandibular disorders: review, criteria, examinations and specifications, critique. J Craniomandib Disord. 1992 Fall;6(4):301-55. [Medline: 1298767]

15. Farrar JT, Young JP Jr, LaMoreaux L, Werth JL, Poole RM. Clinical importance of changes in chronic pain intensity measured on an 11-point numerical pain rating scale. Pain. 2001 Nov;94(2):149-58. [Medline: 11690728] [doi: 10.1016/S0304-3959(01)00349-9]

16. Ahmad M, Hollender L, Anderson Q, Kartha K, Ohrbach R, Truelove EL, John MT, Schiffman EL. Research diagnostic criteria for temporomandibular disorders (RDC/TMD): development of image analysis criteria and examiner reliability for image analysis. Oral Surg Oral Med Oral Pathol Oral Radiol Endod. 2009 Jun;107(6):844-60. [Medline: 19464658] [PMC free article: $\underline{3139469}$ ] [doi: 10.1016/i.tripleo.2009.02.023]

17. Altman DG. Practical statistic for medical research. 1st ed. London: Chapman and Hall; 1991. p. 403-9.

18. Fryback DG, Thornbury JR. The efficacy of diagnostic imaging. Med Decis Making. 1991 Apr-Jun;11(2):88-94. [Medline: 1907710] [doi: 10.1177/0272989X9101100203]

19. Larheim TA. Role of magnetic resonance imaging in the clinical diagnosis of the temporomandibular joint. Cells Tissues Organs. 2005;180(1):6-21. [Medline: 16088129$]$

20. Limchaichana N, Petersson A, Rohlin M. The efficacy of magnetic resonance imaging in the diagnosis of degenerative and inflammatory temporomandibular joint disorders: a systematic literature review. Oral Surg Oral Med Oral Pathol Oral Radiol Endod. 2006 Oct;102(4):521-36. [Medline: 16997121] [doi: 10.1016/j.tripleo.2006.02.001]

21. Honda K, Natsumi Y, Urade M. Correlation between MRI evidence of degenerative condylar surface changes, induction of articular disc displacement and pathological joint sounds in the temporomandibular joint. Gerodontology. 2008 Dec;25(4):251-7. [Medline: 18312371] [doi: 10.1111/j.1741-2358.2008.00219.x]

22. Akerman S, Kopp S, Rohlin M. Histological changes in temporomandibular joints from elderly individuals. An autopsy study. Acta Odontol Scand. 1986 Aug;44(4):231-9. [Medline: 3465195] [doi: 10.3109/00016358608997725]

\section{To cite this article:}

Ekberg E, Hansson LG, List T, Eriksson L, Sahlström LE, Petersson A. Can MRI Observations Predict Treatment Outcome of Lavage in Patients with Painful TMJ Disc Displacement without Reduction?

J Oral Maxillofac Res 2015;6(1):e5

URL: http://www.ejomr.org/JOMR/archives/2015/1/e5/v6n1e5.pdf

doi: $10.5037 /$ jomr.2015.6105 
Copyright (C) Ekberg E, Hansson LG, List T, Eriksson L, Sahlström LE, Petersson A. Published in the JOURNAL OF ORAL \& MAXILLOFACIAL RESEARCH (http://www.ejomr.org), 31 March 2015.

This is an open-access article, first published in the JOURNAL OF ORAL \& MAXILLOFACIAL RESEARCH, distributed under the terms of the Creative Commons Attribution-Noncommercial-No Derivative Works 3.0 Unported License, which permits unrestricted non-commercial use, distribution, and reproduction in any medium, provided the original work and is properly cited. The copyright, license information and link to the original publication on (http://www.ejomr.org) must be included. 